\title{
APLICAÇÃO DE MÉTODOS QUIMIOMÉTRICOS NA ESPECIAÇÃO DE Cr(VI) EM SOLUÇÃO AQUOSA
}

\author{
Marcelo M. Sena, Carol H. Collins*, Kenneth E. Collins
}

Instituto de Química, Universidade Estadual de Campinas, CP 6154, 13083-970 Campinas - SP

Ieda S. Scarminio

Departamento de Química, Universidade Estadual de Londrina, CP 6001, 86051-970 Londrina - PR

\begin{abstract}
CHEMOMETRIC METHODS APPLIED TO THE SPECIATION OF AQUEOUS Cr(VI) SOLUTIONS. The equilibria, the spectra and the identities of the species of $\mathrm{Cr}(\mathrm{VI})$ that are present in aqueous solution have long been an active subject of discussion in the literature. In this paper, three different chemometric methodologies are applied to sets of UV/Visible spectra of aqueous $\mathrm{Cr}(\mathrm{VI})$ solutions, in order to solve a chemical system where there is no available information concerning the composition of the samples nor spectral information about the pure species. Imbrie Q-mode factor analysis, followed by varimax rotation and Imbrie oblique projection, were used to estimate the composition of $\mathrm{Cr}(\mathrm{VI})$ equilibrium solutions and, by combining these results with the k-matrix method, to obtain the pure spectra of the species. Evolving factor analysis and self modeling curve resolution were used to confirm the number of the species and the resolution of the system, respectively. Sets of $3.3 \times 10^{-4}$ and $3.3 \times 10^{-5} \mathrm{~mol} \mathrm{~L}^{-1} \mathrm{Cr}(\mathrm{VI})$ solutions, respectively, were analyzed in the $\mathrm{pH}$ range from 1 to 12 . Two factors were identified, which were related to the chromate ion $\left(\mathrm{CrO}_{4}{ }^{2-}\right)$ and bichromate ion $\left(\mathrm{HCrO}_{4}^{-}\right)$. The $\mathrm{pK}$ of the equilibrium was estimated as 5.8.
\end{abstract}

Keywords: chromium(VI); chemometrics; Imbrie Q-mode factor analysis; evolving factor analysis; self modeling curve resolution; equilibrium $\mathrm{pK}$.

\section{INTRODUÇÃO}

\section{A Controvérsia sobre as Espécies de $\operatorname{Cr}(\mathrm{VI})$}

A química inorgânica do crômio é rica em cores, geometrias e estados de oxidação. Seus estados de oxidação vão de -2 até +6 . Os estados +2 e +3 são os mais estáveis termodinamicamente, enquanto +3 e +6 são os de maior importância em solução aquosa. $\mathrm{O}$ estudo do comportamento químico do $\mathrm{Cr}(\mathrm{VI})$ em solução aquosa é de especial importância devido a vários fatores. Ele é amplamente utilizado como agente oxidante seletivo para substratos orgânicos ${ }^{1}$, as suas soluções ácidas são usadas como padrões de absorção na região do UV/Visíviel ${ }^{2,3}$ e como padrões para dosimetria de radiação ${ }^{4}$. Além disso, as espécies de $\operatorname{Cr}(\mathrm{VI})$ são consideradas carcinogênicas ${ }^{5,6}$ devido à sua natureza fortemente oxidante e à sua mobilidade através de membranas animais, enquanto a redução do $\mathrm{Cr}(\mathrm{VI})$ a $\mathrm{Cr}(\mathrm{III})$ desempenha um importante papel no impacto ambiental do crômio ${ }^{6,7}$.

$\mathrm{O} \mathrm{Cr}(\mathrm{VI})$ existe apenas na forma de oxo-espécies e é rapidamente hidrolisado em solução aquosa, gerando espécies neutras ou aniônicas. As principais espécies de $\mathrm{Cr}(\mathrm{VI})$ presumivelmente presentes em soluções aquosas são os íons bicromato $\left(\mathrm{HCrO}_{4}^{-}\right)$, cromato $\left(\mathrm{CrO}_{4}{ }^{2-}\right)$ e dicromato $\left(\mathrm{Cr}_{2} \mathrm{O}_{7}{ }^{2-}\right)$, além de acido crômico $\left(\mathrm{H}_{2} \mathrm{CrO}_{4}\right)$, que existe em meio ácido concentrado. Os equilíbrios químicos mais estudados envolvendo o Cr(VI) são:

$\mathrm{H}_{2} \mathrm{CrO}_{4} \rightleftharpoons \mathrm{HCrO}_{4}^{-}+\mathrm{H}^{+}$

$\mathrm{HCrO}_{4}^{-} \rightleftharpoons \mathrm{CrO}_{4}{ }^{2-}+\mathrm{H}^{+}$

$2 \mathrm{HCrO}_{4}^{-} \rightleftharpoons \mathrm{Cr}_{2} \mathrm{O}_{7}^{2-}+\mathrm{H}_{2} \mathrm{O}$

$2 \mathrm{CrO}_{4}^{-}+2 \mathrm{H}^{+} \rightleftharpoons \mathrm{Cr}_{2} \mathrm{O}_{7}^{2-}+\mathrm{H}_{2} \mathrm{O}$

Baes e Mesmer ${ }^{8}$, considerando estas equações, indicam que

e-mail: chc@iqm.unicamp.br as concentrações relativas das espécies de $\mathrm{Cr}(\mathrm{VI})$ dependem da concentração total de $\mathrm{Cr}(\mathrm{VI})$ e da acidez da solução. Por outro lado, ainda existe controvérsia na literatura sobre as espécies de $\mathrm{Cr}(\mathrm{VI})$ presentes em solução aquosa, os seus espectros e os valores das constantes de equilíbrio envolvidas. Por exemplo, nos anos 80, Michel et al. ${ }^{9-10}$, analisando espectros Raman, indicavam que $\mathrm{CrO}_{4}{ }^{2-}$ e $\mathrm{Cr}_{2} \mathrm{O}_{7}{ }^{2-}$ eram as únicas espécies de $\mathrm{Cr}(\mathrm{VI})$ presentes em solução aquosa, negando a existência de espécies protonadas. Estudos recentes, como o de Poulopoulou et al. ${ }^{11}$, analisando espectros no UV/visível, continuam questionando a presença da espécie $\mathrm{HCrO}_{4}^{-}$em soluções aquosas. Entretanto, muitos trabalhos contradizem esta hipótese e reafirmam a existência e a importância das espécies protonadas de $\mathrm{Cr}(\mathrm{VI})^{12-18}$. Outro aspecto que causa polêmica diz respeito às faixas de predominância das espécies em função da concentração total de $\mathrm{Cr}(\mathrm{VI})$ e do $\mathrm{pH}^{19-22}$. Valores de constante de equilíbrio para as equações (1) e (2) encontrados na literatura chegam a diferir em mais de uma ordem de grandeza ${ }^{12,13,16-18,21-25}$. De toda esta discussão, é possível propor algumas linhas gerais para a existência e predominância das espécies de $\mathrm{Cr}(\mathrm{VI})^{17,20}: \mathrm{H}_{2} \mathrm{CrO}_{4}$ só deve existir em soluções de $\mathrm{pH}$ abaixo de $0 ; \mathrm{HCrO}_{4}{ }^{-}$deve predominar na faixa de $\mathrm{pH}$ entre 1 e 6 e, acima de uma certa concentração (em torno de $10^{-3} \mathrm{~mol} \mathrm{~L}^{-1}$ ), deve coexistir com $\mathrm{Cr}_{2} \mathrm{O}_{7}{ }^{2-}$; em valores de $\mathrm{pH}$ acima de 8 , deve existir somente $\mathrm{CrO}_{4}{ }^{2-}$. Não obstante, diferenças de opinião em relação a estas generalizações ainda existem na literatura.

\section{A Quimiometria como Ferramenta para a Resolução de Sistemas Multicomponentes}

A Quimiometria, área da química que estuda a aplicação de métodos estatísticos multivariados, se consolidou nos últimos anos, tornando possível a resolução de problemas antes considerados insolúveis. Entre as áreas de maior interesse em quimiometria, estão a calibração e a resolução de sistemas multicomponentes ${ }^{26}$. Uma série de métodos foram desenvolvidos, visando à determinação do número de espécies presentes em misturas, à identificação e à estimativa da concentração dessas espécies. A aplicabilidade de cada método depende da 
informação experimental disponível e do objetivo da análise Problemas envolvendo sistemas multicomponentes em química analítica podem ser classificados em três categorias ${ }^{27}$ : sistemas brancos, para os quais existe informação espectral disponível sobre todas as espécies presentes; sistemas cinzas, para os quais a informação espectral disponível é parcial; e sistemas pretos, para os quais não há qualquer informação disponível a priori sobre as espécies puras. Existe uma série de métodos bem conhecidos para a resolução de sistemas brancos, dos quais os principais são regressão linear múltipla ${ }^{28,29}$ (RLM), filtro de $\mathrm{Kalman}^{30}$, método da matriz $\mathrm{k}^{31,32}$, método da matriz $\mathrm{p}^{32}$, regressão nas componentes principais ${ }^{28,29}$ (PCR, do inglês "principal component regression") e regressão por mínimos quadrados parciais ${ }^{28,29}$ (PLS, "partial least squares"). O método mais usado na resolução de sistemas cinzas é a análise de fatores por aniquilação do posto generalizada ${ }^{33}$ (GRAFA, "generalized rank annihilation factor analysis"). Para a resolução de sistemas pretos, podem ser citados: o método para análise de espectros correlacionados ${ }^{34}$ (DATAN, "data analysis"), a resolução de curvas por auto modelagem ${ }^{35}$ (SMCR, "self-modeling curve resolution"), a análise de fatores evolucionária ${ }^{36,37}$ (EFA, "evolving factor analysis"), a análise multivariada por auto modelagem interativa ${ }^{38,39}$ (SIMPLISMA, "interactive self modeling multivariate analysis"), o método da projeção ortogonal $^{39}$ (OPA, “orthogonal projection approach"), a análise de fatores por faixas usando um alvo teste ${ }^{40}$ (WTTFA, "window target-testing factor analysis") e os métodos multidimensionais, tais como a análise paralela de fatores ${ }^{41,42}$ (PARAFAC, "parallel factor analysis").

Dentre os métodos citados acima os mais comuns são o PLS e a PCR, ambos baseados na decomposição em componentes principais (CPs). A análise de componentes principais ${ }^{29}$ (PCA) é baseada na decomposição dos dados em autovetores e autovalores. Alguns autores ${ }^{43,44}$ apontam uma diferença conceitual entre CPs e fatores: as CPs explicam o máximo de variância contida nas variáveis observadas, enquanto os fatores explicam o máximo de intercorrelação entre as variáveis. No entanto, neste artigo, assim como na maioria da literatura química, ambos os termos serão usados como sinônimos. Existem dois tipos de análise de fatores (AF): tipo $\mathrm{R}$ e tipo $\mathrm{Q}^{43-45}$. Praticamente todas as aplicações de AF encontradas na literatura química são do tipo R. Embora a $\mathrm{AF}$ do tipo Q possa apresentar algumas vantagens em relação à $A F$ do tipo $R$ e seu uso seja comum em áreas correlatas, como a geoquímica ${ }^{46,47}$, a sua aplicação em química praticamente não existe. A AF do tipo Q pode apresentar vantagens computacionais, como cálculos mais rápidos, usando matrizes menores, dependendo do conjunto de dados; e pode também fornecer resultados mais interpretáveis. Na resolução de sistemas brancos, a AF do tipo $\mathrm{Q}$ não requer um conjunto de calibração extenso, como o PLS, a PCR e a RLM, sendo suficientes os espectros puros das espécies absorventes. Na resolução de sistemas pretos, o uso da AF do tipo $\mathrm{Q}$, seguida de rotações, permite determinar o número de componentes e suas concentrações a partir de um conjunto de dados espectrais ${ }^{26}$.

\section{Objetivos}

O objetivo deste artigo é a aplicação de alguns métodos quimiométricos no estudo dos equilíbrios das espécies de $\mathrm{Cr}(\mathrm{VI})$ presentes em solução aquosa, através da análise de espectros no UV/visível, sem nenhum conhecimento prévio dos componentes puros (espectros ou concentrações). Foram aplicados três diferentes métodos quimiométricos. No primeiro, a análise de fatores do tipo Q de Imbrie, seguida de rotação varimax e projeção oblíqua de Imbrie, foi usada para estimar a composição dos equilíbrios. Combinando estes resultados com o método da matriz $\mathrm{k}$, foi possível obter os espectros das espécies puras. No segundo método, a análise de fatores evolucionária foi utilizada na confirmação do número de espécies presentes no sistema e de suas respectivas faixas de existência. No terceiro método, foi utilizada a resolução de curvas por auto modelagem, com a finalidade de comparar os resultados obtidos na primeira parte com um outro método utilizado na resolução de sistemas pretos.

\section{MÉTODOS QUIMIOMÉTRICOS}

\section{Misturas}

Uma mistura é um sistema multicomponente, no qual a soma de todos os componentes permanece constante para cada amostra. A resposta medida para uma mistura é função da proporção entre os componentes e não da quantidade total da mistura. Muitos problemas químicos fornecem conjuntos de dados na forma de misturas. Nesses problemas, os dados originais são normalizados para uma soma constante. Se as respostas para as q espécies puras são conhecidas, o problema pode ser resolvido usando um simplex q-dimensional ${ }^{48}$. Como a soma dos componentes é constante, somente as concentrações de q1 espécies são independentes. Quando não existe informação $a$ priori sobre as respostas das q espécies puras, como no presente caso envolvendo as espécies de $\mathrm{Cr}(\mathrm{VI})$, um método mais robusto é necessário.

\section{Análise de Fatores do Tipo $Q$ de Imbrie, seguida de Rotação Varimax e Projeção Oblíqua de Imbrie, combinada com o Método da Matriz k}

\section{Análise de fatores}

O objetivo da análise de fatores (AF) ${ }^{26,28,43-45}$ é a decomposição de uma matriz de dados $\mathbf{A}_{(\mathrm{n} \times \mathrm{p})}$, formada pelas respostas analíticas de $\mathrm{n}$ objetos (amostras ou misturas) e $\mathrm{p}$ variáveis (tempos de retenção de picos cromatográficos, comprimentos de onda de absorção, etc.) no produto de duas matrizes:

$\mathbf{A}_{(\mathrm{n} \times \mathrm{p})}=\mathbf{P}_{(\mathrm{n} \times \mathrm{q})} \mathbf{T}_{(\mathrm{q} \times \mathrm{p})}^{\mathrm{t}}+\mathbf{E}_{(\mathrm{n} \times \mathrm{p})}$

onde $\mathbf{T}$ é a matriz dos escores dos fatores, $\mathbf{P}$ é a matriz dos pesos ("loadings" em inglês) dos fatores, $\mathbf{E}$ é a matriz dos resíduos, $\mathrm{q}$ indica o número de fatores que descreve a maior parte da variância contida nos dados originais e o sobrescrito t representa a transposta da matriz. Cada fator corresponde a uma coluna da matriz $\mathbf{P}$ e a uma linha correspondente na matriz $\mathbf{T}^{\mathrm{t}}$.

Os dois tipos de AF, tipo $\mathrm{Q}$ e tipo $\mathrm{R}$, se distinguem em função do tipo de escalamento feito antes da decomposição da matriz de dados e em função da maneira pela qual os dados são interpretados. $\mathrm{Na} \mathrm{AF}$ do tipo $\mathrm{R}$, a matriz de dados $\mathbf{A}$ é inicialmente escalada como $\mathbf{A}^{\mathrm{t}} \mathbf{A}$, enquanto na $\mathrm{AF}$ do tipo $\mathrm{Q}$, a ordem muda e $\mathbf{A}$ é escalada como $\mathbf{A} \mathbf{A}^{\mathbf{t}}$. O tipo de escalamento normalmente usado na AF do tipo $\mathrm{R}$ leva a uma matriz de correlação ou covariância. Por outro lado, existem vários tipos de $\mathrm{AF}$ do tipo $\mathrm{Q}$, que dependem de como a matriz de similaridade ou associação é previamente definida. Os três mais comuns são os métodos de Imbrie, de Benzécri (análise de correspondência) e de Gowers ${ }^{43}$.

\section{Análise de fatores do tipo $Q$ de Imbrie}

De uma certa maneira, os escores dos fatores obtidos na AF do tipo $\mathrm{R}$ descrevem as relações entre os objetos. No entanto, a covariância ou a correlação não são os melhores critérios para descrever a similaridade entre objetos, pois são definidas em função das variáveis. A AF do tipo $Q^{26,43,49}$ define a similaridade entre objetos considerando as proporções entre eles. Ela procura os objetos mais distintos na matriz de dados $\mathbf{A}$, os quais são equivalentes as espécies puras ou aos vértices de um simplex. Os demais objetos deverão ser combinações lineares 
deste primeiros. O número mínimo de vértices ou membros terminais é determinado pela aproximação da matriz original por uma matriz de ordem menor, definindo, assim, o número de vetores linearmente independentes e a dimensionalidade do sistema. A composição de cada objeto é obtida por uma análise de autovetores, feita sobre uma matriz real e simétrica, a qual é obtida a partir da matriz de dados originais $\mathbf{A}$.

Imbrie e Purdue ${ }^{43}$ definiram um índice de similaridade chamado $\operatorname{cosseno}$ teta $(\cos \theta)$. Para dois objetos, $\mathrm{n}$ e $\mathrm{m}$, o $\cos \theta$ é definido como

$$
\cos \theta_{n m}=\frac{\sum_{j=1}^{q} a_{n j} a_{m j}}{\sqrt{\sum_{j=1}^{q} a_{n j}^{2} \sum_{j=1}^{q} a_{m j}^{2}}}
$$

onde $a$ é um elemento da matriz A. Para dados positivos, $\cos \theta$ varia entre um, ou seja nenhuma similaridade, e zero, identidade. O procedimento matemático da $\mathrm{AF}$ do tipo $\mathrm{Q}$ de Imbrie inicia-se $\operatorname{com}$ o cálculo dos valores de $\cos \theta$ para todos os pares de objetos da matriz A. A etapa seguinte é a formação da matriz diagonal $\mathbf{D}_{(\mathrm{n} \times \mathrm{n})}$, que contém na sua diagonal principal as raízes quadradas do comprimento de cada vetor linha. Então, $\mathbf{A}_{(\mathrm{n} \times \mathrm{p})}$ é normalizada por linha,

$\mathbf{W}_{(\mathrm{n} \times \mathrm{p})}=\mathbf{D}^{-1} \mathbf{A}$

onde $\mathbf{D}^{-1}$ é a inversa de $\mathbf{D}$. Esta normalização remove o efeito das diferentes proporções entre os objetos, mas não afeta a proporcionalidade entre as variáveis. A seguir, a matriz de similaridade ou associação é calculada como

$\mathbf{H}=\mathbf{W} \mathbf{W}^{\mathrm{t}}=\mathbf{D}^{-1} \mathbf{A} \mathbf{A}^{\mathrm{t}} \mathbf{D}^{-1}$

Esta matriz pode ser expressa aproximadamente como o produto de uma matriz de pesos, $\mathbf{P}_{(\mathrm{n} \times \mathrm{q})}$, e uma matriz de escores, $\mathbf{T}_{(\mathrm{q} \times \mathrm{p})}^{\mathrm{t}}$, onde q é o posto aproximado da matriz $\mathbf{W}$. A matriz $\mathbf{H}$, quadrada e simétrica, pode ser fatorada como $\mathbf{H}=\mathbf{U} \boldsymbol{\Lambda} \mathbf{U}^{\mathrm{t}}$, onde $\mathbf{U}$ é a matriz dos autovetores e $\boldsymbol{\Lambda}$ é a matriz diagonal dos autovalores associados. Assim, $\mathbf{P}=\mathbf{U} \boldsymbol{\Lambda}^{1 / 2} \mathrm{e}$ $\mathrm{T}=\mathbf{W}^{\mathbf{t}} \mathbf{P} \boldsymbol{\Lambda}^{-1}$ podem ser obtidas. Deve-se enfatizar que as matrizes $\mathbf{T}$ e $\mathbf{P}$ obtidas na $\mathrm{AF}$ do tipo $\mathrm{Q}$ são diferentes das matrizes $\mathbf{T}$ e $\mathbf{P}$ obtidas na AF do tipo R.

\section{Rotação varimax}

Após a etapa anterior, é necessária a interpretação da natureza química dos fatores obtidos. Entretanto, a matriz $\mathbf{P}$ obtida na AF do tipo Q de Imbrie é apenas uma possibilidade dentro de uma infinidade de matrizes matematicamente equivalentes. Os eixos dos fatores de $\mathbf{P}$ precisam, portanto, ser rotacionados, buscando uma matriz que tenha significado químico. A rotação varimax $^{26,43,49}$ gira rigidamente os eixos dos fatores, de modo que eles se aproximem o máximo dos vetores mais divergentes no espaço q-dimensional. $\mathrm{O}$ procedimento da rotação varimax maximiza a variância dos pesos dos fatores em cada vetor, sob a restrição de que os fatores permaneçam ortogonais. A matriz $\mathrm{P}$ é rodada, produzindo uma nova matriz, $\mathrm{F}$,

\section{$\mathbf{F}=\mathbf{P R}$}

onde $\mathbf{R}_{(\mathrm{q} \times \mathrm{q})}$ é a matriz de rotação e $\mathbf{F}_{(\mathrm{n} \times \mathrm{q})}$ é a matriz dos pesos dos fatores varimax. Cada linha de $\mathbf{F}$ corresponde a um objeto e cada coluna de $\mathbf{F}$, a um fator.

\section{Projeção oblíqua de Imbrie}

Imbrie $^{26,43,49}$ descreveu uma projeção oblíqua que gira os eixos ortogonais dos fatores varimax, de modo que eles coincidam com os vetores (objetos) mais divergentes no espaço, sem a restrição de que os fatores permaneçam ortogonais. Os outros objetos serão, então, definidos como proporções desses membros terminais. A desvantagem de que os fatores resultantes possuem alguma correlação entre si é compensada pelo fato de que esses fatores correspondem a objetos ou amostras reais. No procedimento, é construída uma matriz $\mathbf{V}_{(\mathrm{q} \times \mathrm{q})}$, que contém, em cada coluna, os maiores valores absolutos dos pesos dos fatores varimax. A matriz de projeção oblíqua, $\mathbf{C}$, é dada por

$\mathbf{C}=\mathbf{F V}^{-1}$

As linhas de $\mathbf{C}$ fornecem a composição de todos os objetos em função dos membros terminais. Finalmente, cada vetor coluna de $\mathbf{C}$ precisa ser desnormalizado, ou seja, dividido pelo comprimento do correspondente objeto original, obtendo-se, assim, a composição final em função dos dados originais.

\section{Método da matriz k}

O método da matriz $\mathrm{k}^{31,32}$ é baseado na Lei de Beer-Lambert,

$\mathbf{A}_{\mathrm{j}}=\sum_{\mathrm{j}=1}^{\mathrm{q}} \mathrm{a}_{\mathrm{ij}} \mathrm{bc} \mathrm{c}_{\mathrm{i}}$

onde $A_{j}$ é a absorbância de uma amostra multicomponente no comprimento de onda $\mathrm{j}, \mathrm{a}_{\mathrm{ij}}$ é a absortividade da espécie i no comprimento de onda $\mathrm{j}$, b é o comprimento do caminho ótico e $c_{i}$ é a concentração da i-ésima espécie (q espécies). Definindo $k_{i j}=a_{i j} b$ e adicionando o erro experimental $e_{j}$, no comprimento de onda $\mathrm{j}$, a seguinte expressão é obtida:

$$
\mathbf{A}_{\mathrm{j}}=\sum_{\mathrm{j}=1}^{\mathrm{q}} \mathrm{k}_{\mathrm{ij}} \mathrm{c}_{\mathrm{i}}+\mathrm{e}_{\mathrm{j}}
$$

Se um conjunto de misturas de composição conhecida é usado na calibração, uma série de equações simultâneas pode ser usada para descrever o espectro da mistura. A equação matricial correspondente é dada por

$$
\mathbf{A}_{(\mathrm{p} \times \mathrm{n})}^{\mathrm{t}}=\mathbf{K}_{(\mathrm{p} \times \mathrm{q})} \mathbf{C}_{(\mathrm{q} \times \mathrm{n})}+\mathbf{E}_{(\mathrm{p} \times \mathrm{n})}
$$

onde $\mathbf{A}$ contém os espectros das amostras, $\mathbf{C}$, a concentração das $\mathrm{q}$ espécies nas $\mathrm{n}$ amostras $(\mathrm{n} \geq \mathrm{q})$, os elementos de $\mathbf{K}$ são estimados por mínimos quadrados e $\mathbf{E}$ é a matriz de erros. Se a Lei de Beer-Lambert for válida, as colunas da matriz $\mathbf{K}$ fornecem os espectros das espécies puras na faixa de comprimento de onda usada na análise, na concentração e comprimento do caminho ótico unitários. Se $\mathbf{C C}^{\mathrm{t}}$ for não singular, a solução dos mínimos quadrados ponderados para a equação (13) é

$\mathbf{K}^{*}=\mathbf{A} \mathbf{C}^{\mathrm{t}}\left(\mathbf{C} \mathbf{C}^{\mathrm{t}}\right)^{-1}$

onde $\mathbf{K}^{*}$ contém os espectros estimados das espécies puras. Deve ser enfatizado que, no método aqui aplicado, $\mathbf{K}^{*}$ é calculada a partir de $\mathbf{C}$, a qual foi obtida após a projeção oblíqua de Imbrie.

\section{Análise de Fatores Evolucionária}

A idéia central da análise de fatores evolucionária (EFA) $)^{36,37}$ é o acompanhamento da evolução do posto de uma matriz de dados $\mathbf{A}$, em função de alguma variação (ex: tempo, pH). O posto define a dimensionalidade da matriz e, portanto, o número de componentes no sistema (fatores). Este acompanhamento é feito pelo exame das submatrizes $\mathbf{A}_{\mathrm{i}}$, de $\mathbf{A}$, formadas pelos primeiros i resultados (espectros ou cromatogramas). A medida que uma nova espécie surge, o posto de $\mathbf{A}$ aumenta de 
uma unidade, o que pode ser acompanhado graficamente. Uma varredura no sentido inverso permite o conhecimento do ponto em que cada espécie desaparece. A combinação destes dois resultados fornece as chamadas janelas de concentração, que são as regiões de existência de cada espécie. A Figura 1 ilustra o acompanhamento do posto em uma eluição cromatográfica com picos sobrepostos.



Figura 1. Evolução do posto de uma matiz de dados coletados em um processo de eluição cromatográfica.

\section{Resolução de Curvas por Auto Modelagem}

A resolução de curvas por auto modelagem $(\mathrm{SMCR})^{35,50}$ é um outro método adequado para a resolução de sistemas pretos. Ele pode ser utilizado na determinação de duas funções contínuas $f_{1}(j)$ e $f_{2}(j)$ a partir de um conjunto de misturas aditivas (combinações lineares) destas mesmas funções. Este tipo de problema é encontrado em dados espectroscópicos e cromatográficos. Na aplicação à espectrofotometria o método considera duas condições: concentrações e espectros não negativos e aditividade dos espectros puros. Portanto, a curva deve ser contínua, combinação linear de dois componentes e positiva em todo o intervalo, ou seja, as funções $f_{1}(j)$ e $f_{2}(j)$ devem ser positivas no intervalo $\left[\mathrm{L}_{1}, \mathrm{~L}_{2}\right]$,

$A(j)=\alpha f_{1}(j)+\beta f_{2}(j), L_{1} \leq j \leq L_{2}$

Outra condição é que não haja em $\left[\mathrm{L}_{1}, \mathrm{~L}_{2}\right]$ pontos em que $\mathrm{f}_{1}(\mathrm{j})$ e $\mathrm{f}_{2}(\mathrm{j})$ sejam simultaneamente iguais a zero. O SMCR considera que as $\mathrm{n}$ amostras são misturas de duas substâncias $\mathrm{X}$ e $\mathrm{Y}$ e que o seu comportamento espectrofométrico segue a Lei de Beer-Lambert:

$A_{i}(j)=x_{i} g_{1}(j)+y_{i} g_{2}(j)$, em $\left[L_{1}, L_{2}\right]$

onde $\mathrm{j}$ é o comprimento de onda, $\mathrm{x}_{\mathrm{i}}$ e $\mathrm{y}_{\mathrm{i}}$ são, respectivamente, as concentrações de $\mathrm{X}$ e $\mathrm{Y}$ na mistura, e $\mathrm{g}_{1}(\mathrm{j})$ e $\mathrm{g}_{2}(\mathrm{j})$ são, respectivamente, os espectros puros de $\mathrm{X}$ e de Y. Como apenas a matriz de dados A é conhecida, uma análise de componentes principais (ou uma AF do tipo R) pode ser aplicada levando a um produto de duas matrizes, uma de escores, T, e uma de pesos, P. A equação obtida é equivalente à equação (5), a qual, descartando-se a matriz de erros, E, torna-se

$$
\mathbf{A}=\mathbf{T P}^{\mathbf{t}}
$$

Portanto, $\mathbf{A}_{\mathbf{i}}(\mathrm{j})=\mathrm{t}_{1 \mathrm{i}} \mathbf{p}_{\mathbf{1}}+\mathrm{t}_{2 \mathrm{i}} \mathbf{p}_{\mathbf{2}}$. Como $\mathbf{P}$ corresponde a uma matriz de vetores linearmente independentes e ortogonais, pode-se dizer que $\mathbf{f}_{1}(\mathrm{j})=\mathrm{n}_{11} \mathbf{p}_{1}+\mathrm{n}_{12} \mathbf{p}_{2}$ é o espectro puro normalizado de $X$, e $\mathbf{f}_{2}(\mathrm{j})=\mathrm{n}_{21} \mathbf{p}_{1}+\mathrm{n}_{22} \mathbf{p}_{2}$ é o espectro puro normalizado de $\mathrm{Y}$. Como os vetores $\mathbf{p}_{\mathbf{1}}$ e $\mathbf{p}_{\mathbf{2}}$ já são conhecidos, é preciso encontrar $\mathrm{n}_{11}, \mathrm{n}_{12}, \mathrm{n}_{21}$ e $\mathrm{n}_{22}$, que correspondem às coordenadas em $\mathbf{T}$. Como $\mathbf{A}(\mathrm{j})$ é sempre positiva, $\mathrm{t}_{1 \mathrm{i}} \mathbf{p}_{\mathbf{1}}+$ $\mathrm{t}_{2 \mathrm{i}} \mathbf{p}_{\mathbf{2}} \geq 0$. Se é admitida a existência de dois comprimentos de onda onde apenas uma espécie absorve, obtém-se:

$\mathrm{t}_{1} \mathbf{p}_{1 \mathbf{a}}+\mathrm{t}_{2} \mathbf{p}_{2 \mathrm{a}}=0($ reta 1$)$

$\mathrm{t}_{1} \mathbf{p}_{1 \mathbf{b}}+\mathrm{t}_{2} \mathbf{p}_{\mathbf{2 b}}=0($ reta 2$)$

Estas duas retas podem ser traçadas em um gráfico de $t_{2} \mathrm{x}$ $\mathrm{t}_{1}$ (Figura 2) e devem conter os escores correspondentes aos espectros puros. O próximo passo é encontrar os espectros normalizados: $; f(j) d j=1$, ou seja, a área sob a curva $f(j)$ é igual a um. A partir disto, é deduzida a equação de uma terceira reta:

$\sum\left(\mathrm{t}_{1} \mathrm{p}_{1}+\mathrm{t}_{2} \mathrm{p}_{2}\right) \delta \mathrm{j}=1$ ( $\delta \mathrm{j}$ é o intervalo entre os comprimentos de onda)

$\sum\left(\mathrm{t}_{1} \mathrm{p}_{1}\right) \delta \mathrm{j}+\sum\left(\mathrm{t}_{2} \mathrm{p}_{2}\right) \delta \mathrm{j}=1$

$\mathrm{t}_{1} \delta \mathrm{j}\left(\sum \mathrm{p}_{1}\right)+\mathrm{t}_{2} \delta \mathrm{j}\left(\sum \mathrm{p}_{2}\right)=1$

$\sum \mathrm{p}_{1}=\mathrm{c}_{1}$ e $\sum \mathrm{p}_{2}=\mathrm{c}_{2}\left(\mathrm{c}_{1}\right.$ e $\mathrm{c}_{2}$ são constantes $)$

$\mathrm{t}_{1} \mathrm{c}_{1}+\mathrm{t}_{2} \mathrm{c}_{2}=1 / \delta \mathrm{j}($ reta 3$)$

Assim, no cruzamento da reta $3 \mathrm{com}$ as retas 1 e 2 obtémse os escores correspondentes aos espectros puros e normalizados, possibilitando a resolução do sistema, como pode ser observado na Figura 2.

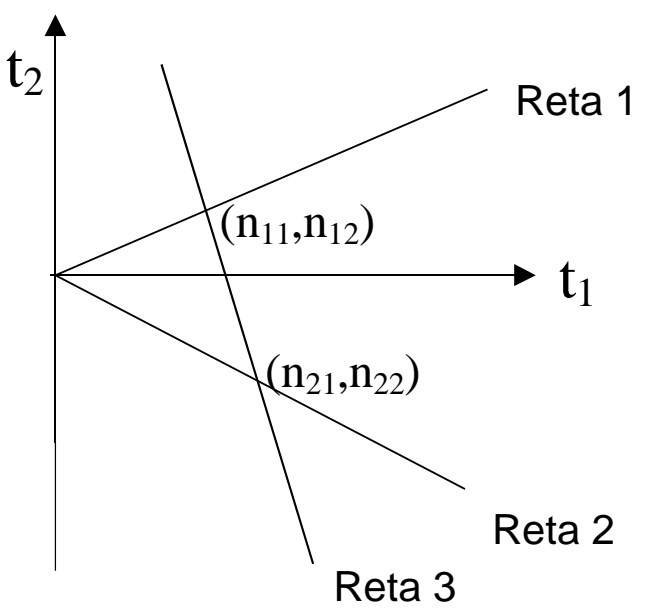

Figura 2. Gráfico dos escores $t_{2} x t_{1}$, mostrando a solução para a resolução de curvas por auto modelagem, a qual é dada pela intersecção da reta 3 com as retas 1 e 2 .

\section{PARTE EXPERIMENTAL}

O hidróxido de sódio, o perclorato de sódio monohidratado e o ácido perclórico concentrado (aproximadamente $70 \%$ ) foram adquiridos da Merck e usados sem purificações prévias. $\mathrm{O}$ dicromato de potássio (Riolab) foi penta-recristalizado e seco em estufa a $70{ }^{\circ} \mathrm{C}$ por dois dias. Uma solução estoque, $3,3 \mathrm{x}$ $10^{-2} \mathrm{~mol} \mathrm{~L}^{-1}$ de $\mathrm{Cr}(\mathrm{VI})$ em água, foi preparada a partir do dicromato e padronizada por titulação com ferro(II), de acordo com um método da literatura ${ }^{51}$. Duas soluções intermediárias, $3,3 \times 10^{-3}$ e $3,3 \times 10^{-4} \mathrm{~mol} \mathrm{~L}^{-1}$ em $\mathrm{Cr}(\mathrm{VI})$, foram preparadas a partir da solução estoque. Outras soluções estoque foram também preparadas: perclorato de sódio $1,00 \mathrm{~mol} \mathrm{~L}^{-1}$, ácido perclórico $1,0 \mathrm{~mol} \mathrm{~L}^{-1}$ e hidróxido de sódio $0,1 \mathrm{~mol} \mathrm{~L}^{-1}$,. Todas as soluções foram preparadas em água deionizada 
(Barnsted). As soluções de ácido e de base foram apropriadamente padronizadas ${ }^{51}$.

Dois conjuntos, cada um com 15 soluções problema de $\mathrm{Cr}(\mathrm{VI})$, nas concentrações de $3,3 \times 10^{-4}$ e $3,3 \times 10^{-5} \mathrm{~mol} \mathrm{~L}^{-1}$, respectivamente, foram preparados na faixa de $\mathrm{pH}$ entre $1 \mathrm{e}$ 12 , em balões volumétricos de $100 \mathrm{~mL}$. $\mathrm{O} \mathrm{pH}$ foi ajustado com ácido perclórico ou hidróxido de sódio, usando um pHmetro Micronal B375 e um eletrodo de vidro combinado. A força iônica destas soluções foi ajustada em $0,50 \mathrm{~mol} \mathrm{~L}^{-1}$ com perclorato de sódio.

Os espectros das soluções foram obtidos em um espectrofotômetro Hewlett Packard HP 8452A, com arranjo de diodos, na região do ultra-violeta/visível, entre 190 e 820 $\mathrm{nm}$ ( $2 \mathrm{~nm}$ de resolução). Todas as medidas foram feitas em uma cubeta de quartzo de $1,00 \mathrm{~cm}$ de caminho ótico, a 25 ${ }^{\circ} \mathrm{C}$. O espectrofotômetro foi acoplado a um microcomputador PC/AT 486 (16 Mb de memória RAM) e o software "General Scanning" foi usado na aquisição dos dados. Os dados foram tratados em um microcomputador Pentium $233 \mathrm{MHz}$ (32 Mb de RAM). Os programas de análise de fatores do tipo Q de Imbrie, rotação varimax, projeção oblíqua e método da matriz $\mathrm{k}$ foram escritos em linguagem FORTRAN, sendo executados em ambiente DOS. O programa de resolução de curvas por auto modelagem foi escrito e executado no software MATLAB, versão 5.2 (The MathWorks, Natick, MA, EUA). O programa de análise de fatores evolucionária foi executado em MATLAB 5.2, utilizado o pacote "PLS toolbox", versão 2.0 (Eigenvector Technologies, Manson, WA, EUA).

\section{RESULTADOS E DISCUSSÃO}

\section{Escolha da Faixa de Comprimento de Onda para Análise}

Da faixa de comprimento de onda original, entre 180 e 320 nm (320 comprimentos de onda), foi selecionada a faixa de 230 a $460 \mathrm{~nm}$ (116 comprimentos de onda) para a análise quimiométrica. A região acima de $460 \mathrm{~nm}$ foi eliminada pois, além de não mostrar qualquer pico relativo ao $\mathrm{Cr}(\mathrm{VI})$, ela pode apresentar uma grande quantidade de ruído. Por outro lado, várias espécies absorvem na região abaixo de $230 \mathrm{~nm}$. Foi observado que a espécie $\mathrm{OH}^{-}$, presente nas soluções básicas, absorve nesta região, adicionando mais um fator significativo aos resultados quimiométricos. Por isso, essa região também foi eliminada.

\section{Análise de Fatores do Tipo $Q$ de Imbrie, seguida de Rotação Varimax e Projeção Oblíqua de Imbrie, combinada com o Método da Matriz k}

Foram analisados dois diferentes conjuntos de soluções de Cr(VI), nas concentrações $3,3 \times 10^{-4}$ e $3,3 \times 10^{-5} \mathrm{~mol} \mathrm{~L}^{-1}$, respectivamente, ambos na faixa de $\mathrm{pH}$ de 1 a 12 . As superfícies espectrais obtidas para esses dois conjuntos são mostradas na Figura 3. O sistema estudado neste artigo é um típico sistema preto, no qual, a priori, as espécies puras não podem ser isoladas quimicamente nem obtidas separadamente. A maior dificuldade deste tipo de análise é a definição do número de fatores que deve ser usado, visando determinar o número de espécies presentes no sistema.

A análise de fatores do tipo Q de Imbrie, seguida de rotações, dos dois conjuntos de soluções apresentou resultados bastante similares. Em ambos os casos, o número de fatores selecionados para o modelo foi igual a dois, os quais foram atribuídos as espécies bicromato $\left(\mathrm{HCrO}_{4}{ }^{-}\right)$e cromato $\left(\mathrm{CrO}_{4}{ }^{2-}\right)$, respectivamente. As atribuições foram feitas com base nos perfis de $\mathrm{pH}$ estimados e nos espectros das espécies obtidos em seguida, através do método da matriz $\mathrm{k}$. As estimativas da distribuição das espécies em função do $\mathrm{pH}$, para os conjuntos de
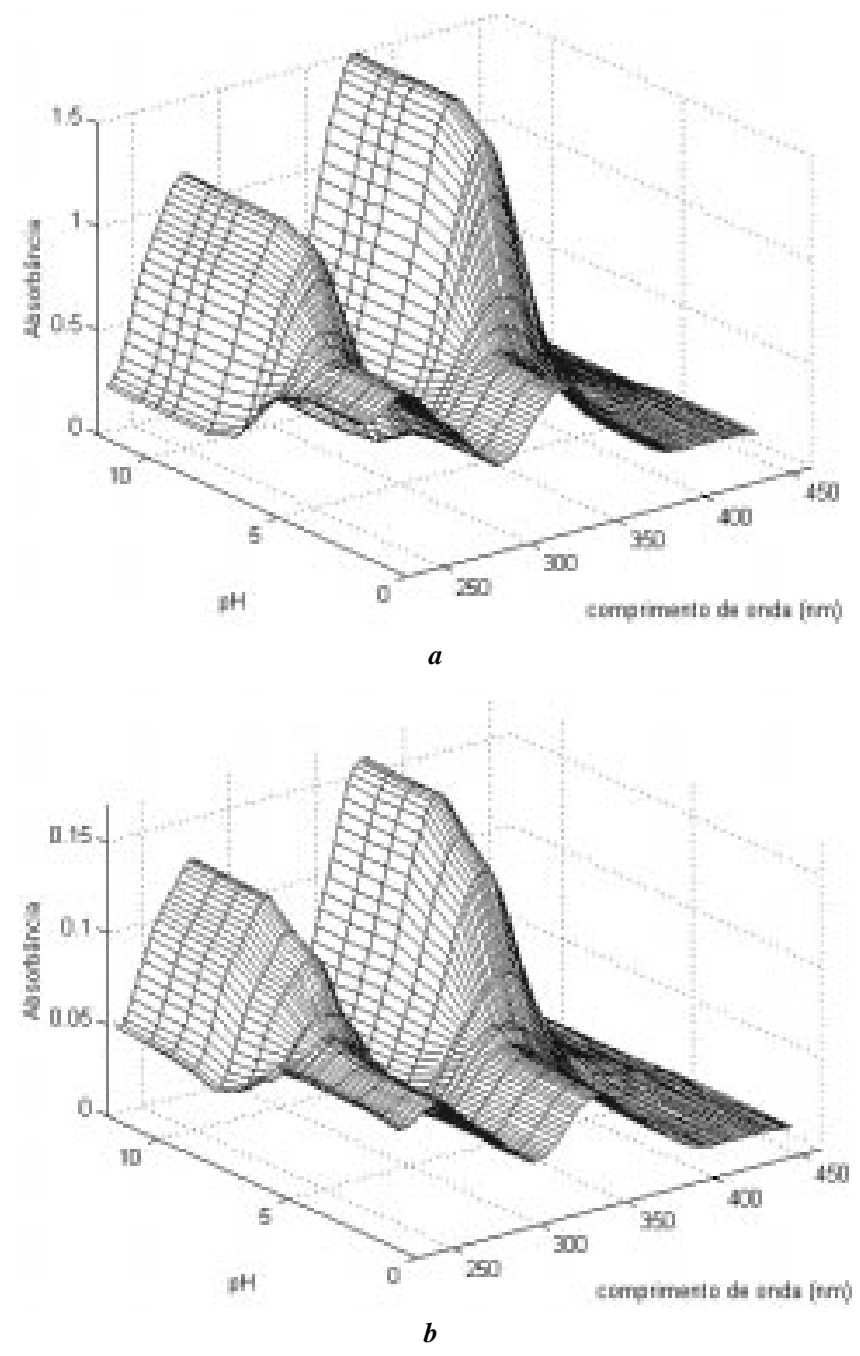

Figura 3. Superfícies espectrais obtidas para os conjuntos de soluções (a) $3,3 \times 10^{-4} \mathrm{~mol} \mathrm{~L}^{-1} \mathrm{e}(\boldsymbol{b}) 3,3 \times 10^{-5} \mathrm{~mol} \mathrm{~L}^{-1} \mathrm{em} \mathrm{Cr}(\mathrm{VI})$, ambas na faixa de $\mathrm{pH}$ de 1 a 12.

soluções $3,3 \times 10^{-4}$ e $3,3 \times 10^{-5} \mathrm{~mol} \mathrm{~L}^{-1}$ em $\mathrm{Cr}(\mathrm{VI})$, são mostradas na Figura 4. O modelo selecionado para o conjunto de soluções $3,3 \times 10^{-4} \mathrm{~mol} \mathrm{~L}^{-1}$ em $\mathrm{Cr}(\mathrm{VI})$, após as rotações, explicou $99,96 \%$ (fator $1=94,34 \%$ e fator $2=5,62 \%$ ) da variância total dos dados originais (Figura 4a). O modelo selecionado para o conjunto de soluções $3,3 \times 10^{-5} \mathrm{~mol} \mathrm{~L}^{-1} \mathrm{em}$ $\mathrm{Cr}(\mathrm{VI})$ explicou $99,97 \%$ (fator $1=95,91 \%$ e fator $2=4,06 \%$ ) da variância total (Figura $4 \mathrm{~b}$ ).

Os perfis de $\mathrm{pH}$ obtidos para a distribuição bicromato-cromato estão em boa concordância com a literatura ${ }^{8,16-18,20-22,49,52}$, levando em conta que, na concentração de $\mathrm{Cr}(\mathrm{VI})$ analisada, menor que $3,3 \times 10^{-4}$ mol L ${ }^{-1}$, a presença da espécie dicromato pode ser considerada insignificante ${ }^{8,19-22}$. A constante de dissociação para o íon bicromato pode ser estimada através do cruzamento das linhas na Figura 4. O valor de pK estimado para o equilíbrio bicromato-cromato em solução aquosa $\left(I=0,5 \mathrm{~mol} \mathrm{~L}^{-1}\right)$ foi igual a 5,8 na Figura $4 \mathrm{a}$ e a 5,9 na Figura 4b (o erro de estimativa do gráfico é de aproximadamente 0,1 unidades de $\mathrm{pK}$ ). Valores de $\mathrm{pK}$ encontrados na literatura para este equilíbrio variam desde 5,8 até 7,8 12,16-18,21-25,49. Entretanto, os valores de pK estimados mais recentemente estão bastante próximos dos encontrados neste trabalho: 6,09 (determinações espectrofotométrica e potenciométrica, [Cr(VI)] entre 0,1 e $1,92 \times 10^{-3} \mathrm{~mol} \mathrm{~L}^{-1}$, $\mathrm{I}=0,1 \mathrm{~mol} \mathrm{~L}^{-1}, 25^{\circ} \mathrm{C}$ ) ${ }^{16} ; 5,89$ (determinação potenciométrica, $[\mathrm{Cr}(\mathrm{VI})]$ entre 25 e $100 \times 10^{-3} \mathrm{~mol} \mathrm{~L}^{-1}, \mathrm{I}=3,0 \mathrm{~mol} \mathrm{~L}^{-1}$, 


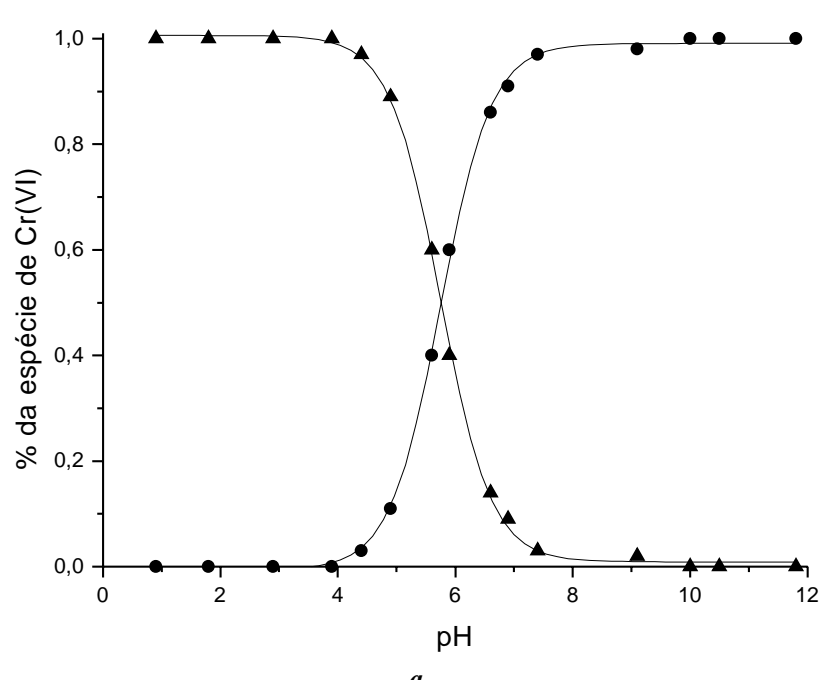

$\boldsymbol{a}$

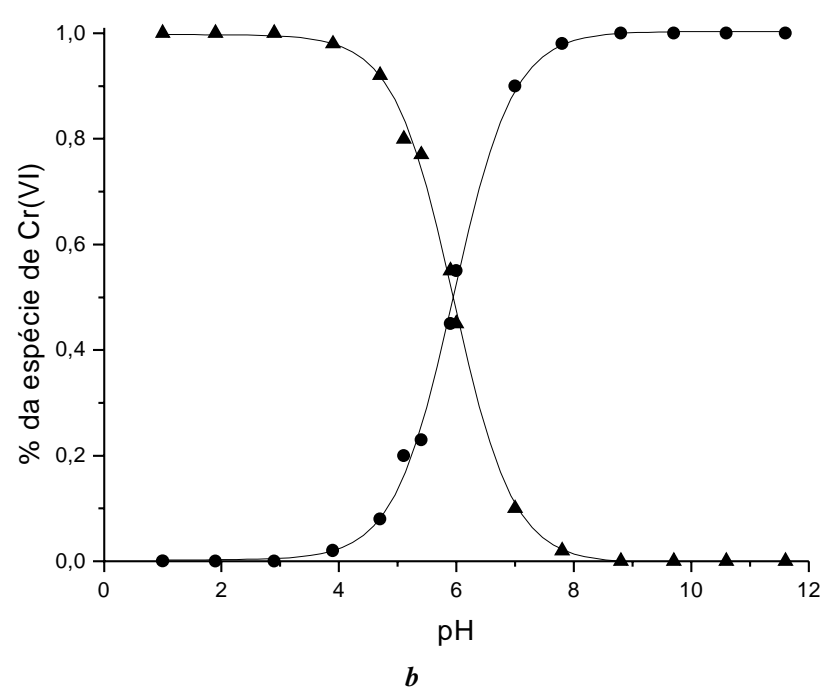

b

Figura 4. Perfis de distribuição das espécies de Cr(VI) em função do $p H$, estimados pela análise de fatores do tipo $Q$ de Imbrie, seguida de rotações. (a) $[\mathrm{Cr}(\mathrm{VI})]=3,3 \times 10^{-4} \mathrm{~mol} \mathrm{~L}^{-1}$ e (b) $[\mathrm{Cr}(\mathrm{VI})]=3,3 \times 10^{-5}$

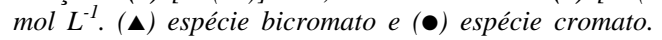

$\left.25^{\circ} \mathrm{C}\right)^{17}$ e 6,01 (determinação a partir de espectros de Raman, $[\mathrm{Cr}(\mathrm{VI})]$ entre 12 e $\left.18 \times 10^{-3} \mathrm{~mol} \mathrm{~L}^{-1}, \mathrm{I}=0,1 \mathrm{~mol} \mathrm{~L}^{-1}, 25^{\circ} \mathrm{C}\right)^{18}$.

Os espectros das duas espécies puras puderam ser estimados pelo método da matriz $\mathrm{k}$, usando como entrada os pesos dos fatores obtidos após a projeção oblíqua. Os espectros estimados para as espécies bicromato e cromato foram idênti$\cos$ em ambos os conjuntos e são mostrados na Figura 5. Estes espectros são muito semelhantes aos relatados por vários autores ${ }^{12,16,53}$.

\section{Análise de Fatores Evolucionária}

A análise de fatores evolucionária foi aplicada aos dois conjuntos de dados, visando a confirmação do número de espécies presentes e de suas respectivas faixas de existência. O resultado para o conjunto de $[\mathrm{Cr}(\mathrm{VI})]=3,3 \times 10^{-4}$ mol L ${ }^{-1}$ é mostrado na Figura 6, na qual os autovalores ou valores singulares são colocados no gráfico em função do $\mathrm{pH}$ das amostras. A observação desta figura permite confirmar a existência de dois valores singulares significativos e, portanto, a presença de apenas duas espécies no sistema. A análise direta (Figura 6a) mostra a presença inicial de uma espécie $\left(\mathrm{HCrO}_{4}^{-}\right)$e o surgimento de uma segunda espécie acima de $\mathrm{pH}_{4}\left(\mathrm{CrO}_{4}{ }^{2-}\right)$. A análise inversa (Figura 6b)



Figura 5. Espectros das espécies puras estimados através do método da matriz $k .(\Delta)$ espécie bicromato e (•) espécie cromato.

mostra que uma espécie $\left(\mathrm{CrO}_{4}{ }^{2-}\right)$ está presente até o final da análise $(\mathrm{pH} 12)$ e a outra $\left(\mathrm{HCrO}_{4}{ }^{-}\right)$desaparece abaixo de $\mathrm{pH}$ 8. Estes resultados confirmam aqueles obtidos na AF do tipo Q de Imbrie, seguida de rotações, com relação as faixas de existência (janelas de $\mathrm{pH}$ ) das espécies bicromato e cromato. Os resultados da análise de fatores evolucionária para o conjunto de $[\mathrm{Cr}(\mathrm{VI})]=3,3 \times 10^{-5} \mathrm{~mol} \mathrm{~L}^{-1}$ foram semelhantes.

\section{Resolução de Curvas por Auto Modelagem}

A resolução de curvas por auto modelagem (SMCR) é um outro método adequado à resolução de sistemas pretos, porém é limitado a presença de apenas duas espécies. Como já foi constatado que o sistema estudado aqui possui apenas duas espécies, o SMCR se mostra adequado para sua resolução. A primeira tentativa de resolução deste sistema, contudo, não foi bem sucedida. Foi analisada a matriz original de dados, contendo as amostras em função do $\mathrm{pH}$ nas linhas e os comprimentos de onda de absorção nas colunas (15 amostras x 116 variáveis). Parecem não existir nas curvas dos espectros das espécies puras, as chamadas variáveis puras, condição necessária para o SMCR poder resolver o sistema, que são variáveis em que a absorção de uma espécie seja zero enquanto a absorção da outra seja significativa. A análise do conjunto de soluções $3,3 \times 10^{-4} \mathrm{~mol} \mathrm{~L}^{-1} \mathrm{em}$ $\mathrm{Cr}$ (VI) levou aos espectros mostrados na Figura 7. O espectro associado à espécie $\mathrm{CrO}_{4}{ }^{2-}$ é bastante semelhante ao que era esperado. Por outro lado, o espectro associado à espécie $\mathrm{HCrO}_{4}^{-}$é bem diferente do obtido na metodologia anterior (método da matriz k). Observando-se na Figura 5 os espectros puros esperados, é possível entender porque a deconvolução não foi bem sucedida neste caso. No espectro do $\mathrm{CrO}_{4}{ }^{2-}$ existe um ponto (variável pura), no vale próximo a $315 \mathrm{~nm}$, onde a absorção do $\mathrm{CrO}_{4}{ }^{2-}$ é nula e a absorção da outra espécie é significativa. Já para o espectro do $\mathrm{HCrO}_{4}^{-}$ (Figura 5), não existe tal ponto. $\mathrm{O}$ espectro do $\mathrm{HCrO}_{4}{ }^{-} \mathrm{ob}-$ tido na Figura 7 apresenta absorbância zero em aproximadamente $390 \mathrm{~nm}$, que foi o ponto tomado como variável pura, onde a proporção entre as absorções dos espectros das duas espécies é maior.

A resolução do sistema foi possível graças a uma estratégia simples. Ao invés de se analisar a matriz de dados original, analisou-se a matriz de dados transposta. (116 variáveis x 15 amostras). Neste caso, a matriz de pesos ( 2 x 19) obtida na análise de componentes principais (primeiro passo do SMCR) representará as curvas de concentração em função do $\mathrm{pH}$, ao invés dos espectros, como no caso anterior (naquele caso, obtinha-se uma matriz de pesos de dimensões 2 x 116). Pode-se supor que as condições de que as funções sejam contínuas e 

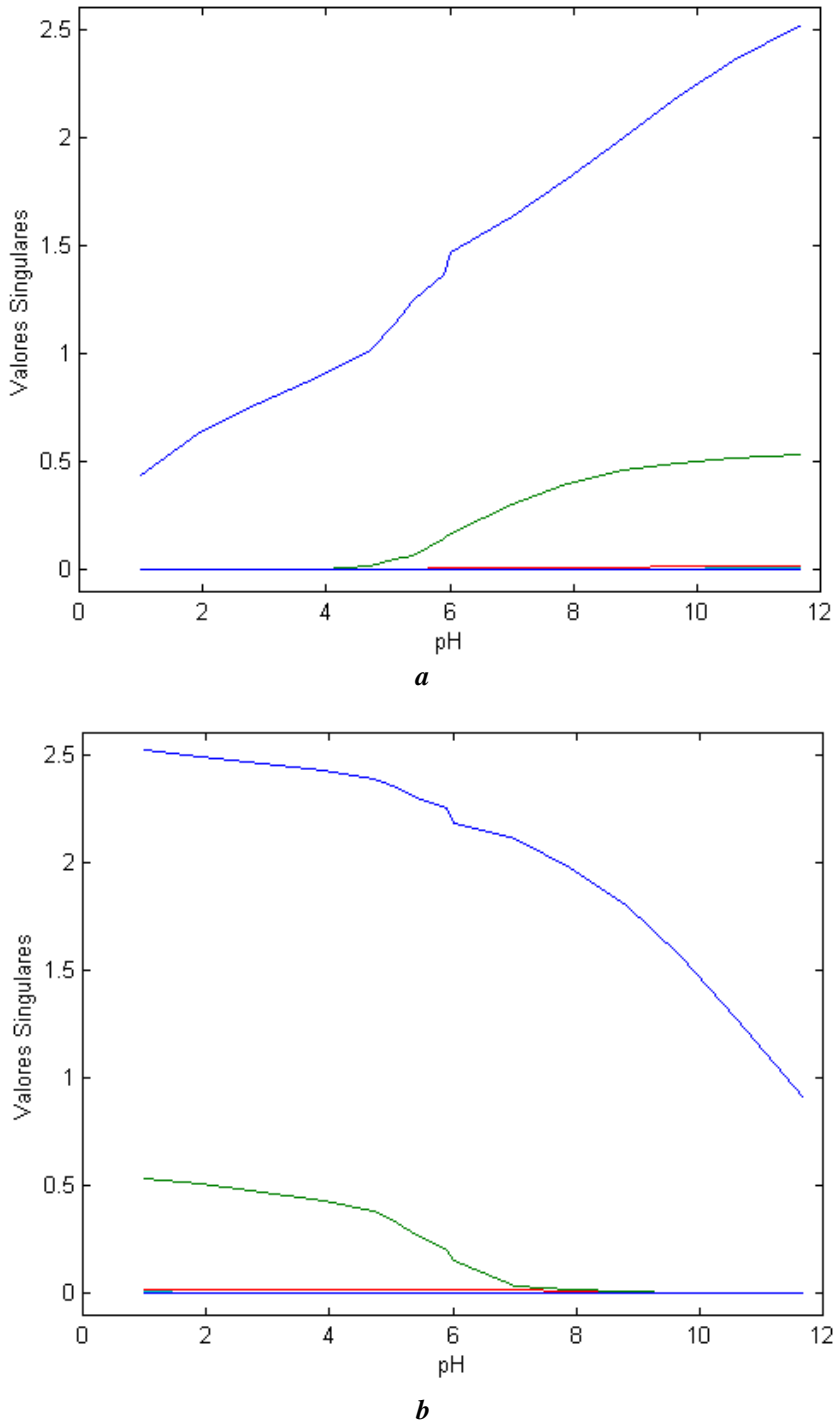

Figura 6. Análise de fatores evolucionária, mostrando os valores singulares em função do $\mathrm{pH}$ da amostra. (a) análise direta; (b) análise inversa. $[\mathrm{Cr}(\mathrm{VI})]=3,3 \times 10^{-4} \mathrm{~mol} \mathrm{~L} L^{-1}$.

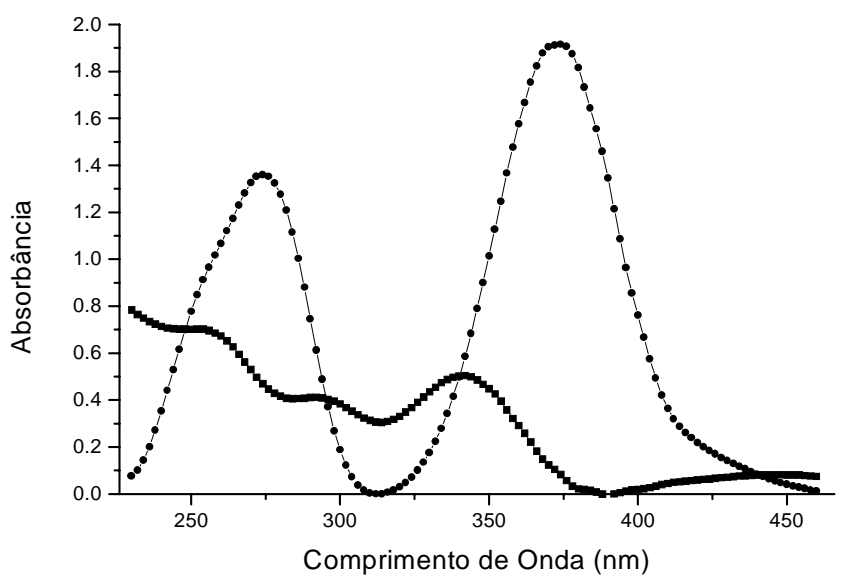

Figura 7. Tentativa malsucedida de deconvolução dos espectros das espécies $(\mathbf{\square})$ bicromato e $(\bullet)$ cromato, utilizando a resolução de curvas por auto modelagem. $[\mathrm{Cr}(\mathrm{VI})]=3,3 \times 10^{-4} \mathrm{~mol} \mathrm{~L}^{-1}$.

possuam variáveis puras, cada uma, serão satisfeitas neste caso. Com isso, foi possível a resolução do sistema, sendo estimados o perfil da distribuição das espécies de $\mathrm{Cr}(\mathrm{VI})$ em função do $\mathrm{pH}$ (Figura 8a) e os espectros das espécies puras (Figura 8b). Ambos os resultados são praticamente iguais aos obtidos na AF do tipo Q de Imbrie, seguida de rotações, e combinada com o método da matriz $\mathrm{k}$. O valor de $\mathrm{pK}$ para o equilíbrio entre as espécies bicromato e cromato, 5,8, obtido pela intersecção das curvas na Figura 8a, também é idêntico ao que foi estimado na primeira parte deste trabalho. A análise do conjunto de soluções $3,3 \times 10^{-5} \mathrm{~mol} \mathrm{~L}^{-1}$ em $\mathrm{Cr}(\mathrm{VI})$ apresentou resultados praticamente iguais aos do primeiro conjunto.
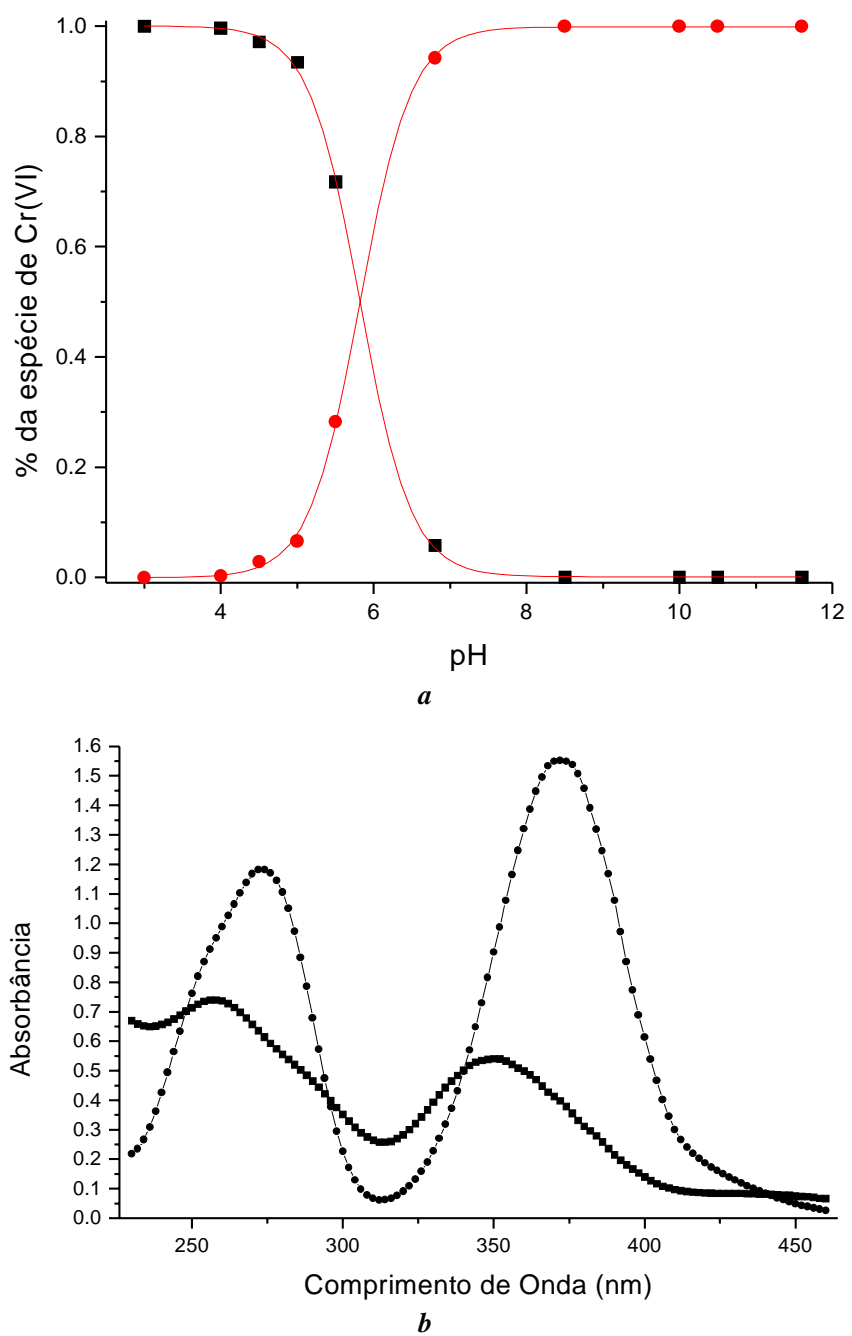

Figura 8. Resolução do sistema de soluções $3,3 \times 10^{-4} \mathrm{~mol} \mathrm{~L}^{-1}$ em $\mathrm{Cr}(V I)$, utilizando a resolução de curvas por auto modelagem. (a) perfil de distribuição das espécies em função do $\mathrm{pH}$ e (b) espectros das espécies puras. (ロ) bicromato e $(\bullet)$ cromato.

\section{CONCLUSÃO}

$\mathrm{O}$ aspecto mais relevante deste artigo é a aplicação de métodos quimiométricos na resolução de sistemas pretos, que são sistemas químicos para os quais não existe a priori informação disponível relativa à composição ou aos espectros das espécies puras. Os métodos utilizados apresentam, entre outras vantagens, a não necessidade do uso de um conjunto de calibração para a determinação da composição das amostras. A AF do tipo $\mathrm{Q}$ apresenta, ainda, várias vantagens sobre a AF do tipo $\mathrm{R}$, mais tradicionalmente utilizada em aplicações químicas. Nos passos intermediários da presente aplicação, matrizes $15 \times 15$ foram diagonalizadas, ao invés de matrizes $116 \times 116$ no caso do tipo R. Na análise de espectros com um grande número de 
comprimentos de onda, a AF do tipo $\mathrm{Q}$ pode chegar a requerer um tempo computacional significativamente menor para os cálculos. Além disso, não é necessário o uso de regressão na determinação das concentrações, e a presença de amostras anômalas ("outliers") não interfere nos resultados das outras amostras.

Um outro aspecto é a utilização da espectrofotômetria de UV/Visível combinada com as metodologias quimiométricas, como uma nova maneira de estimar valores de $\mathrm{pK}$, que pode ser aplicada a ácidos ou bases muito fracas. Este artigo também contribuiu para o estudo dos polêmicos equilíbrios das espécies de $\mathrm{Cr}(\mathrm{VI})$ em solução aquosa, confirmando a existência de espécies protonadas e estimando um valor de $\mathrm{pK}$ para a o equilíbrio bicromato-cromato que está em concordância com a literatura mais recente ${ }^{16-18}$.

No presente trabalho, três diferentes métodos quimiométricos foram aplicados ao mesmo conjunto de dados, sendo que os melhores resultados foram obtidos com a $\mathrm{AF}$ do tipo $\mathrm{Q}$ de Imbrie, seguida de rotações e combinada com o método da matriz k. A análise de fatores evolucionária confirmou a presença de apenas duas espécies, enquanto a resolução de curvas por auto modelagem é menos robusta e só conseguiu resolver as curvas, após a aplicação de uma estratégia de manuseio dos dados. Finalmente, o uso da quimiometria possibilita a solução de problemas que seriam insolúveis pelos métodos convencionais, tais como a resolução de equilíbrios complexos e a determinação de constantes cinéticas para reações onde ocorre sobreposição espectral entre as espécies.

\section{AGRADECIMENTOS}

Os autores agradecem ao $\mathrm{CNPq}$ pelo suporte financeiro, ao doutorando Marlon M. dos Reis e à Prof ${ }^{\mathrm{a}}$. Drª . Márcia M. C. Ferreira (IQ - UNICAMP) pelas sugestões e implementação do programa de resolução de curvas por auto modelagem, ao Prof. Dr. Ronei J. Poppi (IQ - UNICAMP) pelas sugestões em relação à análise de fatores evolucionária e ao Prof. Dr. Sérgio H. Pezzin (Faculdade de Engenharia de Joinville - UESC) pelas valiosas discussões sobre o $\mathrm{Cr}(\mathrm{VI})$.

\section{REFERÊNCIAS}

1. Colton, R.; Coordin. Chem. Rev. 1987, 78, 1.

2. Burke, R. W.; Deardorff, E. R.; Menis, O.; J. Res. Natl. Bur. Stand. 1972, 76A, 469.

3. Gil, M.; Escolar, D.; Iza, N.; Montero, J. L.; Appl. Spectrosc. 1986, 40, 1156.

4. Sharpe, P. H. G.; Barrett, J. H.; Berkley, A. M.; Int. J. Appl. Radiat. Isot. 1985, 36, 647.

5. Casadevall, M.; Fresco, P. D.; Kortenkamp, A.; Chem.Biol. Interact. 1999, 123, 117.

6. Kimbrough, D. E.; Cohen, Y.; Winer, A. M.; Creelman, L.; Mabuni, C.; Crit. Rev. Environ. Sci. Tec. 1999, 29, 1.

7. Harzdorf, A. C.; Int. J. Environ. Anal. Chem. 1987, 29, 249.

8. Baes, C. F.; Mesmer, R. E.; The Hydrolysis of Cations; John Wiley \& Sons; New York, 1976, p. 211-218.

9. Michel, G.; Machiroux, R.; J. Raman Spectrosc. 1983, 14, 22.

10. Michel, G.; Cahay, R.; J. Raman Spectrosc. 1986, 17, 79.

11. Poulopoulou, V. G.; Vrachnou, E.; Koinis, S.; Katakis, D.; Polyhedron 1997, 16, 521.

12. Bailey, N.; Carrington, A.; Lott, K. A. K.; Symons, M. C. R.; J. Chem. Soc. 1960, 290.

13. Haight Jr., G. P.; Richardson, D. C.; Coburn, N. H.; Inorg. Chem. 1964, 3, 1777.

14. Baharad, R.; Hayman, B. P.; Wolff, M. A.; J. Phys. Chem. 1969, 73, 4391.

15. Lukkari, O.; Suom. Kemi. 1970, 43B, 347.

16. Cruywagen, J. J.; Heyns, J. B. B.; Rohwer, E.A.; Polyhedron 1998, 17, 1741.
17. Brito, F.; Ascanio, J.; Mateo, S.; Hernandez, C.; Araujo, L.; Gili, P.; MartinZarza, P.; Dominguez, S.; Mederos, A.; Polyhedron 1997, 16, 3835.

18. Heyns, J. B. B.; Cruywagen, J. J.; Carron, K. T.; J. Raman Spectrosc. 1999, 30, 335.

19. Tandon, R. K.; Crisp, P. T.; Ellis, J.; Baker, R. S.; Talanta 1984, 31, 227.

20. Tong, S.; Li, K.; Talanta 1986, 331, 775.

21. Kondratenko, N. A.; Sherstyuk, V. P.; Theor. Exp. Chem. 1986, 22, 656.

22. Lugo Rivera, J. L.; Tese de Doutorado, Instituto de Química, UNICAMP, Campinas, SP, 1997.

23. Linge, H. G.; Jones, A. L.; Aust. J. Chem. 1968, 21, 2189.

24. Vasilev, V. P.; Raskova, O. G.; Vasileva, V. N.; Golykh, N. V.; Russ. J. Inorg. Chem. 1981, 26, 212.

25. Palmer, D. A.; Wesolowski, D.; Mesmer, R. E.; J. Solution Chem. 1987, 16, 443.

26. Scarminio, I. S.; Ishikawa, D. N.; Barreto, W. J.; Paczkowski, E. D.; Arruda, I.C.; Quim. Nova 1998, $21,590$.

27. Liang, Y. Z.; Kvalheim, O. M.; Manne, R.; Chemom. Intell. Lab. Syst. 1993, 18, 235.

28. Beebe, K. R.; Kowalski, B. R.; Anal. Chem. 1987, 59, 1007A.

29. Martens, H.; Naes, T.; Multivariate Calibration; John Wiley \& Sons; New York, 1989.

30. Andrew, K. N.; Rutan, S. C.; Worsfold, P. J.; Anal. Chim. Acta 1999, 388, 315.

31. Brown, C. W.; Lynch, P. F.; Obremski, R. J.; Lavery, D. S.; Anal. Chem. 1982, 54, 1472.

32. Haaland, D. M.; Easterling, G.; Vopicka, D. A.; Appl. Spectrosc. 1985, 39, 73.

33. Sanchez, E.; Kowalski, B. R.; J. Chemometr. 1988, $2,265$.

34. Scarminio, I. S.; Kubista, M.; Anal. Chem. 1993, 65, 409.

35. Lawton, W. H.; Sylvestre E. A.; Technometrics 1971, $13,617$.

36. Maeder, M.; Anal. Chem. 1987, 59, 527.

37. Maeder, M.; Zilian, A.; Chemom. Intell. Lab. Syst. 1988, $3,205$.

38. Windig, W.; Heckler, C. E.; Agblevor, F. A.; Evans, R. J.; Chemom. Intell. Lab. Syst. 1992, 14, 195.

39. de Braekeler, K.; Massart, D. L.; Chemom. Intell. Lab. Syst. 1997, 39, 127.

40. Lohnes, M. T.; Guy, R. D.; Wentzell, P. D.; Anal. Chim. Acta 1999, 389, 95.

41. Bro, R.; Chemom. Intell. Lab. Syst. 1997, 38, 149.

42. Sena, M. M.; Fernandes, J. C. B.; Rover Jr., L.; Poppi, R. J.; Kubota, L. T.; Anal. Chim. Acta 2000, 409, 159.

43. Jöreskog, K. G.; Klovan, J. E.; Reyment, R. A.; Geological Factor Analysis; Elsevier; Amsterdam, 1976.

44. Paatero, P.; Tapper, U.; Chemom. Intell. Lab. Syst. 1993, $18,183$.

45. Hopke, P. K.; Chemom. Intell. Lab. Syst. 1989, 6, 7.

46. Irino, T.; Tada, R.; Geochem. J. 2000, 34, 59.

47. Aboul Kassin, T. A. T.; Simoneit, B. R. T.; Mar. Chem. 1996, 54, 135.

48. Deane, J. M.; MacFie, H. J. H.; King, A. G.; J. Chemometr. 1989, 3, 359.

49. Sena, M. M.; Dissertação de Mestrado, Instituto de Química, UNICAMP, Campinas, SP, 1996.

50. Biloti, D. N.; dos Reis, M. M.; Ferreira, M. M. C.; Pessine, F. B. T.; J. Mol. Struct. 1999, 481, 557.

51. Basset, J.; Denney, R. C.; Jeffery, G. H.; Mendham, J.; Vogel. Análise Inorgânica Quantitativa; $4^{\mathrm{a}}$ ed., Guanabara; Rio de Janeiro, 1981, p. 268.

52. Cresser, M.S.; Hargitt, R.; Talanta 1976, 23, 153.

53. Davies, W.G.; Prue, J.E.; Trans. Faraday Soc. 1955, 51,1045 